\title{
The Role of Non-alcoholic Fatty Liver Disease in Cardiovascular Disease
}

\author{
Sven M Francque
}

Department of Gastroenterology Hepatology, University Hospital Antwerp \& Laboratory of Experimental Medicine and Paediatrics, Division of Gastroenterology Hepatology, Faculty of Medicine and Health Sciences, University of Antwerp, Antwerp, Belgium

\begin{abstract}
Non-alcoholic fatty liver disease (NAFLD) has become the most prevalent chronic liver disease in western countries and is closely related to the metabolic syndrome. When NAFLD is associated with hepatocellular damage and inflammation (non-alcoholic steatohepatitis [NASH]) it can lead to severe liver disease. However, it has become clear that NAFLD is also associated with an increased risk of cardiovascular disease (CVD), independently of classical known risk factors for the latter. In the current review we briefly summarise the current clinical evidence on the role of NAFLD in CVD and discuss the potential mechanisms by which NAFLD can be linked to the pathophysiology of CVD.
\end{abstract}

\section{Keywords}

non-alcoholic fatty liver disease, steatosis, cardiovascular events, pathophysiology

Disclosure: The author has no conflicts of interest to declare.

Received: 19 May 2014 Accepted: 20 June 2014 Citation: European Cardiology Review, 2014;9(1):10-5

Correspondence: Sven M Francque, Department of Gastroenterology Hepatology, University Hospital Antwerp, Wilrijkstraat 10, 2650 Edegem, Antwerp, Belgium.

E: Sven.francque@uza.be

Non-alcoholic fatty liver disease (NAFLD) has become the most prevalent chronic liver disease in western countries. ${ }^{1,2}$ It is closely associated with obesity, diabetes, dyslipidaemia and the metabolic syndrome, and shares common risk factors and pathophysiological mechanisms with these entities. ${ }^{2,3}$ NAFLD can be associated with hepatocellular damage and inflammation and is then called non-alcoholic steatohepatitis (NASH). ${ }^{4}$ Other than the associated liver-related morbidity and mortality, it has become clear that NAFLD is also associated with an increased risk of cardiovascular disease (CVD). The link between NAFLD and CVD can in part be explained by the common risk factors that they share. However, evidence is increasing that NAFLD is an aetiological factor contributing to the development of CVD, independently of classical known risk factors for the latter. ${ }^{5,6}$ In this review we briefly summarise the current clinical evidence on the role of NAFLD in CVD and discuss the potential mechanisms by which NAFLD can be linked to the pathophysiology of CVD.

\section{Non-alcoholic Fatty Liver Disease}

Steatosis is defined by the accumulation of fat in the hepatocytes. Other than in cases of mitochondrial toxicity (e.g. acute fatty liver of pregnancy, Reye's syndrome) where bipolar lipids accumulate in micelles (often called microvesicular steatosis), ${ }^{8}$ steatosis mainly consists of fat-filled vacuoles delineated by a bi-layer lipid membrane and predominantly accumulating triglycerides. ${ }^{9}$ The fat vacuoles may differ in size, from small vesicles up to large vacuoles that fill the cytoplasm and displace the nucleus towards the border of the cell. This results in the term macrovesicular steatosis.,10 If smaller vacuoles are present, the term mesovesicular is often used. If the dimensions of the vacuoles vary, steatosis is often called mixed type steatosis, although this implies the presence of typical fat vacuoles of varying diameter, and not necessarily the concomitant presence of macrovesicular steatosis and micelles as seen in acute mitochondrial toxicity. ${ }^{8}$

Steatosis can be secondary to several causes, such as the use of alcohol and certain drugs (e.g. furadantin, methotrexate, amiodarone, corticosteroids) to chronic hepatitis C (especially genotype 3 ). ${ }^{4}$ In the absence of these causes, steatosis is called NAFLD.7.11 NAFLD thus not only necessitates the absence of significant alcohol use (defined as $>20 \mathrm{~g} /$ day in women and $>30 \mathrm{~g} /$ day in men) but also the exclusion of all other causes of secondary steatosis, making the term NAFLD not an ideal denominator of this entity..$^{11,12}$ The term has, however, been widely adopted.

NAFLD comprises a wide spectrum of histological liver lesions. If steatosis is the only histological abnormality, it is called non-alcoholic fatty liver (NAFL). ${ }^{12}$ However, if steatosis is accompanied by inflammation and signs of hepatocyte degeneration (ballooning or swelling of hepatocytes because of cytoskeleton damage), it is called NASH. ${ }^{4,7}$ $\mathrm{NASH}$ requires the combination of steatosis, lobular inflammation (portal inflammation is not included in the diagnosis) and ballooning of any degree.,12 These features can only be reliably assessed by liver histology, implying NASH to be a histological diagnosis. 10,12,13 NAFLD can be accompanied by fibrosis, which can progress to cirrhosis and its inherent complications. It is generally accepted that the risk of fibrosis and progression to cirrhosis is confined to patients with NASH (but fibrosis is not part of the definition of NASH), whereas NAFL is believed to run a benign course, at least in terms of liver disease. 10,14,15 Typical features of NASH and even steatosis can disappear in the cirrhotic stage, making it difficult to establish an aetiological diagnosis in some cases of cryptogenic cirrhosis, part of which are considered burned-out NASH based on the presence of metabolic risk factors for NAFLD and NASH. ${ }^{14}$ 
Despite extensive research conducted so far, the pathophysiology of NAFLD and NASH remains poorly understood. Epidemiologically, NAFLD and NASH are closely related to obesity, to dyslipidaemia, to disturbances of glycaemic control and to the metabolic syndrome (MetS). ${ }^{16}$ Therefore, NAFLD is sometimes considered as the hepatic manifestation of the MetS.,37 For some time NAFLD was considered to be secondary to these metabolic features, but insight is growing in the aetiological role of NAFLD in the development of the MetS or type 2 diabetes mellitus (T2DM), which it often precedes. ${ }^{17,18}$ Currently the pathophysiology of NASH is considered to be a parallel multi-hit process involving nutrients, the gut microbiome, the intestinal barrier, the adipose tissue (producing adipokines), the immune system and the liver, together with genetic and environmental factors. ${ }^{19}$

Although exact data vary because of differences in modes of diagnosis and selection of patients, NAFLD seems to affect about 15-30\% of the adult population in western countries, making it the most prevalent liver disease worldwide.1,2 The prevalence of $\mathrm{NASH}$ is estimated at $2-5 \%$, with progressive fibrosis in about $45-50 \%$, with a risk of ultimately developing cirrhosis in 10-20\%., ${ }^{2,15}$ The risk of hepatocellular carcinoma (HCC) is not well-defined, and some concern has risen about the risk of HCC in non-cirrhotic NAFLD. ${ }^{20,21}$

\section{Non-alcoholic Fatty Liver Disease and Cardiovascular Disease}

Whereas the liver-related morbidity and mortality related to NAFLD/ NASH are well-documented and well-known, the consequences of NAFLD outside the setting of liver disease has long been unrecognised but gains growing attention. As already mentioned, NAFLD sometimes precedes the development of T2DM or the MetS, suggesting NAFLD is not simply a consequence but also a causal factor (and probably both) in their pathophysiology. ${ }^{5,17,18}$

Data are accumulating that patients affected by NAFLD have a higher risk of developing cardiovascular (CV) abnormalities, clinical CV events and even CV death. ${ }^{5,22} \mathrm{~A}$ first specific challenge in the interpretation of these data on the link between CVD and NAFLD is to distinguish between a timely correlation simply based on underlying risk factors that are shared by both conditions, or an independent contribution of NAFLD (after correction for these shared metabolic risk factors) in the subsequent development of CVD. The latter implies a specific pathophysiological contribution of the liver affected by NAFLD to the development of CV abnormalities. Elucidating the role of NAFLD in the development of CVD therefore constitutes a second challenge, in which, besides clinical data, studies in animal models might be helpful. Finally the question whether the role of NAFLD in the development of CVD is confined to NASH or is already present in NAFL needs to be answered. This question is particularly relevant for the treatment of NAFLD. If indeed the development of CVD is substantially influenced by NAFLD and NASH, its prevention might constitute an indication to treat NAFLD and its subtypes.

\section{Clinical Data}

The most convincing data on the role of NAFLD in CVD are those on the link between NAFLD and subclinical coronary heart disease (CHD). NAFLD, mostly diagnosed by ultrasound, has been shown to be an independent risk factor for the presence or future development of increased intima-media thickness, impaired flow-mediated vasodilatation, the presence of carotid atherosclerotic plaques, an increased coronary artery calcium score on cardiac computed tomography and abnormal coronary flow reserve as a marker for impaired coronary microcirculation, both in crosssectional and in follow-up studies, after correction for classical risk factors for $\mathrm{CHD} .{ }^{23-36}$

For clinical CHD, data are also emerging from large cohorts of patients, both cross-sectional and longitudinal studies, in community-based cohorts and in more selected patient groups (e.g. patients with T2DM, type 1 diabetes, patients undergoing coronary angiography or patients with documented NAFLD), that NAFLD is an independent predictor for clinical CHD, being the severity of the atherosclerotic lesions on coronarography or the occurrence of fatal and non-fatal CHD events. ${ }^{37-41}$ These data have been extensively reviewed elsewhere. ${ }^{5,6}$ Only a few studies did not confirm the independent relationship of NAFLD with incident CHD or showed it to be confined to patients with NAFLD who concomitantly met the diagnosis of the MetS..$^{42,43}$ Overall the data strongly support the independent contribution of NAFLD to an increased risk of clinically relevant $\mathrm{CHD}$, even after correction for an extended set of well-established risk factors for CHD.

Several studies also showed a link between NAFLD and alterations in cardiac metabolism, ${ }^{35,44}$ structure and haemodynamic function, such as myocardial insulin resistance and mitochondrial adenosine triphosphate (ATP) production, cardiac steatosis, myocardial hypertrophy and left ventricular diastolic dysfunction, not attributable to concomitant diabetes, obesity or arterial hypertension. ${ }^{44-50}$ The severity of these cardiac abnormalities correlated with the severity of the NAFLD. Finally NAFLD has been associated with an increased risk of autonomic dysfunction and cardiac arrhythmias (mainly atrial fibrillation). ${ }^{51-53}$ Interestingly, recent data have shown that NAFLD is also independently linked with QTc interval prolongation, a major risk factor for ventricular arrhythmias and sudden cardiac death, which might explain in part the increased CV mortality associated with NAFLD. ${ }^{54}$ Finally, congestive heart failure and aortic valve sclerosis have also been linked with NAFLD independently of known risk factors. ${ }^{55-57}$

Overall, although not all data are methodologically solid and most of the studies lack a diagnosis by the gold standard, the concept of NAFLD as being an independent contributor to the development of atherosclerosis and other functional and structural CV alterations, which subsequently lead to clinical CVD, seems sufficiently substantiated by the current evidence to integrate it in the clinical approach of both the NAFLD patient and the patient with CVD.

\section{Pathophysiological Considerations}

The mechanisms by which NAFLD influences the development of atherosclerosis and CVD is incompletely understood. NAFLD, T2DM, the MetS and CVD share many metabolic features and risk factors, leading to the concept that they belong to a complex multisystem disease with several organ manifestations and a complex interplay between the different entities, with multiple bidirectional cause-effect relationships. The specific contribution of one entity to the others is therefore difficult to discern, and there might be substantial inter-individual variability.

The contribution of NAFLD to CVD, seen as a unidirectional cause-effect relationship, can be either indirect or direct - the potential mechanisms are summarised in Figure 1. Firstly, as the liver is a key organ in both glucose and lipid homeostasis, it is not surprising that evidence is accumulating that NAFLD plays a role 
Figure 1: Schematic Overview of the Mechanisms that may Link Indirectly or Directly the Liver Affected by Non-alcoholic Fatty Liver Disease to Alterations in the Cardiovascular System

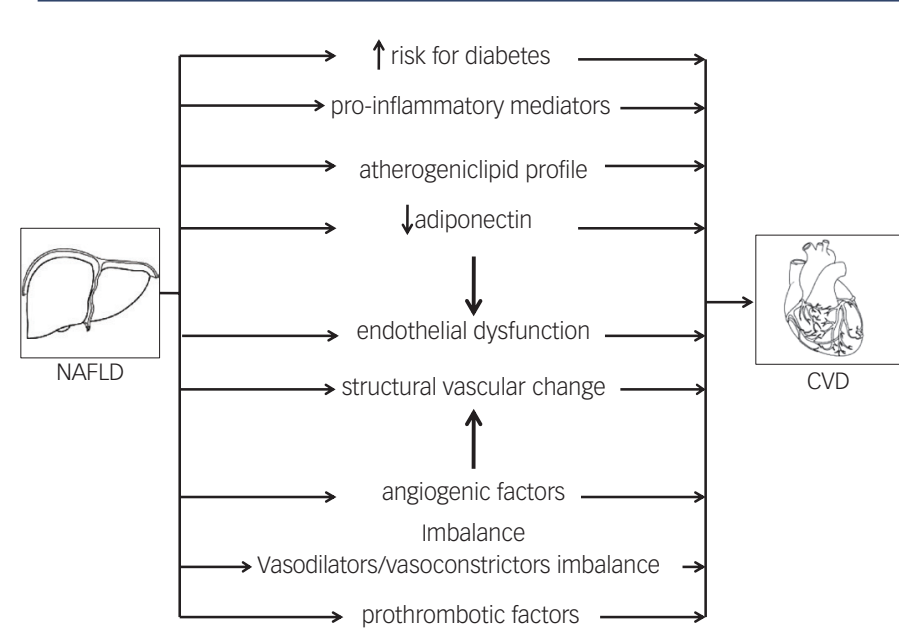

$C V D=$ cardiovascular disease; $N A F L D=$ non-alcoholic fatty liver disease

in the development of T2DM and the MetS, which are by themselves risk factors for CVD. 5,17,18 This links NAFLD only indirectly to CVD.

NAFLD has indeed been shown to contribute to the development of T2DM. Several studies, mostly diagnosing NAFLD by ultrasound or liver enzymes, have shown that NAFLD precedes and predicts the future development of T2DM independent of obesity and other factors of the MetS. ${ }^{58,59}$ As insulin suppresses hepatic gluconeogenesis, NAFLD-associated hepatic insulin resistance results in mild hyperglycaemia, with a need for an increased insulin production to suppress hepatic glucose output and keep it within normal ranges. If Beta ( $\beta$ ) cells cannot sustain this increased insulin secretion, patients develop impaired glucose tolerance and diabetes. Inflammatory mediators released by the inflamed liver in NASH might accelerate this process. ${ }^{60}$

Secondly, the liver might also contribute directly to the development of CVD. It is clear that NAFLD is associated with an atherogenic lipid profile. ${ }^{11}$ In NAFLD, production of triglyceride-rich very-low-density lipoprotein (VLDL) particles is increased. ${ }^{62}$ Insulin normally inhibits adipose tissue lipolysis (which is the main source of free fatty acids flux to the liver for incorporation in hepatic triglycerides) and hepatic VLDL secretion, both of which are hence increased in association with hepatic and adipose tissue insulin resistance. ${ }^{17,63}$ Subsequently, high-density lipoprotein cholesterol lowers and small dense low-density lipoprotein particles increase. Both conditions are highly atherogenic.

Endothelial dysfunction has been shown to be an early event in the development of atherosclerosis. ${ }^{64,65}$ Several studies have recently highlighted that insulin resistance at the endothelial level occurs early in the development of NAFLD and is already present after a few days of high-fat feeding, when steatosis develops but inflammation seems to still be absent. ${ }^{66-68}$ In the endothelium, insulin stimulates nitric oxide (NO) release leading to vasodilatation, and an impairment of insulin signaling leads to a reduced vasodilatory response to acetylcholine (ACh), which is used as a well-established hallmark of endothelial dysfunction. ${ }^{6}$ Steatosis leads to impaired endothelial NO synthase (eNOS) phosphorylation and hence impaired NO response to insulin, contributing to an increase in intrahepatic resistance. ${ }^{66}$ Conversely, insulin-sensitizing drugs improve endothelial function in NAFLD, as demonstrated by an improvement in the vasodilatory response to ACh. ${ }^{66}$ These changes occur early in the development of NAFLD, before the development of inflammation and before peripheral insulin resistance can be documented. ${ }^{68}$ Although the exact mechanisms need to be further elucidated, these findings point towards a pivotal role of steatosis in the impairment of endothelial function as a primary event preceding extrahepatic events.

The increased intrahepatic resistance is not only attributable to endothelial dysfunction based on reduced NO production because of endothelial cell insulin resistance. An imbalance in locally produced vasodilators and vasoconstrictors has also been documented. ${ }^{68}$ Steatosis was shown to be associated with a disturbed production of endothelin 1 and of cyclooxygenase-mediated vasoactive prostaglandins. Although data in humans are scarce and mainly restricted to the measurement of metabolites of vasoactive substances in peripheral blood (both in cirrhosis and in NAFLD patients), alterations have been documented and reflect potential systemic effects of what happens inside a liver affected by NAFLD, and hence its contribution to the development of CV alterations. ${ }^{69}$

Furthermore, steatosis also induces structural abnormalities of liver vasculature that also contributes to the associated increase in intrahepatic resistance. ${ }^{68}$ The pathophysiology of these structural alterations is currently unknown. Angiogenic factors have been shown to play a role in the intrahepatic vascular changes in cirrhosis and are also studied in NASH. ${ }^{70}$ Altered levels of angiogenic factors (vascular endothelial growth factor and its soluble receptors 1 and 2) have also been documented in the peripheral blood of patients with NASH. ${ }^{69}$ This is used as an argument to support the hypothesis of the role of angiogenic factors in the pathophysiology of NASH, but it also might help us to understand the link between the liver and CVD. Although this has not been proven so far, it can be hypothesized that the altered concentrations of angiogenic factors exert their effects in the extrahepatic vascular beds. The role of angiogenic factors, which not only influence vascular growth but also have vasoactive properties in the pathogenesis of atherosclerosis, has been well-documented. ${ }^{71}$

Prothrombotic factors have also shown to play a role in the progression of liver disease. ${ }^{72}$ Several metabolic risk factors are prothrombotic, ${ }^{73}$ but the role of the liver in this prothrombotic state has been poorly documented. Increased levels of prothrombotic factors have been described in patients with NASH. ${ }^{74}$ Although the liver is the main source of most of these coagulation factors, the causal role of the liver has not been proven. We studied an extensive panel of coagulation factors in a large series of histologically proven NAFLD patients, showing that mainly plasminogen activator inhibitor 1 (PAl-1) is increased in association with $\mathrm{NASH}$, whereas some of the other factors (e.g. factor VIII, protein S) are elevated in relation with metabolic parameters, as was shown previously ${ }^{73,75}$ but the alterations of the latter do not correlate with liver histology. ${ }^{74}$ Furthermore, PAl-1 was also elevated in relation to the severity of liver histology. Together these findings point towards an independent contribution of NAFLD severity to a prothrombotic state that might contribute to CVD.

Adiponectin is another factor that might represent a link between NAFLD and CVD. Adiponectin, secreted by adipocytes, is decreased in obesity ${ }^{76,77}$ but also in NAFLD in relation to the histological severity 
Table 1: Prospective Patient-based Cohort Studies on the Risk of Coronary Heart Dsease in Relation to Non-alcoholic Fatty Liver Disease Diagnosed by Liver Histology

\begin{tabular}{|c|c|c|c|c|c|c|c|}
\hline Reference & $\begin{array}{l}\text { Year of } \\
\text { Publication }\end{array}$ & $n$ & $\begin{array}{l}\text { Mean } \\
\text { Follow-up (y) }\end{array}$ & $\begin{array}{l}\text { Histological } \\
\text { Subtypes }\end{array}$ & Comparator & Conclusion & Remark \\
\hline Matteoni et al. ${ }^{92}$ & 1999 & 132 & 18.0 & Different subtypes & $\begin{array}{l}4 \text { histological } \\
\text { subtypes within } \\
\text { the cohort }\end{array}$ & $\begin{array}{l}\text { All-cause and CV mortality } \\
\text { not different between } \\
\text { histological subtypes }\end{array}$ & $\begin{array}{l}\text { Increased liver-related } \\
\text { mortality }\end{array}$ \\
\hline Dam-Larsen et al. ${ }^{93}$ & 2004 & 109 & 16.7 & NAFL & General population & $\begin{array}{l}\text { All-cause and CV } \\
\text { mortality not different }\end{array}$ & - \\
\hline Adams et al. ${ }^{94}$ & 2005 & 420 & 7.6 & NAFLD & General population & $\begin{array}{l}\text { Increased all-cause } \\
\text { mortality }\end{array}$ & $\begin{array}{l}\text { CHD second cause } \\
\text { of death }\end{array}$ \\
\hline Ekstedt et al. ${ }^{95}$ & 2006 & 129 & 13.7 & NAFL/NASH & Reference population & $\begin{array}{l}\text { Increased liver-related } \\
\text { and CV mortality in NASH }\end{array}$ & $\begin{array}{l}\text { NAFL not significantly } \\
\text { different from } \\
\text { reference population }\end{array}$ \\
\hline Rafiq et al. ${ }^{96}$ & 2009 & 173 & 13.0 & NAFL/NASH & NAFL versus NASH & $\begin{array}{l}\text { CHD first cause of } \\
\text { death in both } \\
\text { NAFL and NASH }\end{array}$ & $\begin{array}{l}\text { Increased liver-related } \\
\text { mortality in NASH } \\
\text { compared with NAFL }\end{array}$ \\
\hline Söderberg et al. ${ }^{97}$ & 2010 & 118 & 24.0 & NAFL/NASH & $\begin{array}{l}\text { NAFL versus NASH } \\
\text { versus general } \\
\text { population }\end{array}$ & $\begin{array}{l}\text { Increased CV mortality in } \\
\text { NASH compared with NAFL } \\
\text { and general population }\end{array}$ & $\begin{array}{l}\text { No difference between } \\
\text { NAFL and general } \\
\text { population }\end{array}$ \\
\hline
\end{tabular}

$C H D=$ coronary heart disease; $C V=$ cardiovascular; $n=$ number of patients; $N A F L=$ non-alcoholic fatty liver; NAFLD = non-alcoholic fatty liver disease;

$\mathrm{NASH}=$ non-alcoholic steatohepatitis; $y=$ year.

of NAFLD after correction for body mass index (BMI). ${ }^{78,79}$ Adiponectin has insulin-sensitizing, anti-inflammatory and anti-atherogenic properties $^{80}$ and directly affects endothelial function by eNOS messenger RNA (mRNA) stabilisation and eNOS phosphorylation. ${ }^{81}$ Furthermore, adiponectin stimulates circulating angiogenic cells. ${ }^{82}$ NAFLD-associated adiponectin decrease might therefore contribute to the development of CVD.

Inflammatory mediators can also contribute to the increased risk of CVD. NASH is associated with an increased intrahepatic production of pro-inflammatory cytokines, which are also increased systemically. ${ }^{83-85}$ One of the cytokines that are increased in NASH is Interleukin 6 (IL6), which stimulates angiotensin II in vascular smooth muscle cells with an associated production of reactive oxygen species, which in turn interact with NO production and activity. ${ }^{69}$ Other inflammatory mediators released by the liver might also contribute to atherogenesis.

Although all these mechanisms are plausible links between the liver affected by NAFLD and the development of CVD, no studies to date have scientifically proven to really represent a cause-effect relationship. Several mechanisms are most probably concomitantly present, and might substantially differ between patients. Further study is hence needed to gain mechanistic insight into the pathophysiology of the NAFLD-CVD axis, with an individualised approach, both preventive and therapeutic, as the ultimate goal.

Non-alcoholic Fatty Liver or Non-alcoholic Steatohepatitis? The question whether the role of NAFLD in the development of CVD is confined to NASH or is already present in NAFL is important. Only about $5-10 \%$ of NAFLD patients have $\mathrm{NASH}^{2}{ }^{2}$ so if the risk were to be confined to NASH, this would substantially reduce the CVD burden attributable to NAFLD. This might be in contrast with the current data on the impact of NAFLD on CVD, which does not seem to fit with the relatively small number of NASH patients within the NAFLD group. The answer to this question has potential implications for the management of NAFLD patients. Indeed, if not only NASH but also
NAFL were to be associated with an increased risk of CVD, one might argue that NAFLD should be treated regardless of the presence of NASH. NAFL would then turn out not to be a benign condition, as it still is generally regarded nowadays, ,2,1,12,15 and guidelines for the treatment of NAFLD might have to consider treating NAFL, with prevention of CVD as treatment indication.

However, the question remains largely unanswered. The main reason is that most of the data come from studies where NAFLD is diagnosed based on ultrasound or on liver biochemistry or both. ${ }^{5,6}$ In these studies no distinction is made between NAFL and NASH. This distinction still requires a biopsy. ${ }^{13}$ Series including histology have smaller patient numbers and patients are usually more selected, leading to a potential overrepresentation of more severe liver disease compared with the general population. Furthermore, most of these studies have rather short mean follow-up times. The methodological limitations of these studies hamper the general applicability of their results.

Nevertheless, several data give an indication that the risk is confined to NASH, or is at least higher in NASH patients compared with NAFL. A first indication comes from the studies using liver biochemistry to diagnose NASH. Although it has been well-established that transaminases and gamma-glutamyl transpeptidase (GGT) are not perfectly correlated to the severity of the liver lesions, and that transaminases can be normal in patients with $\mathrm{NASH}^{79}$ as well as NASH can be present in patients with normal transaminases, ${ }^{86}$ overall liver enzymes are higher in NASH versus NAFL patients. ${ }^{79,87}$ Moreover, several scoring systems for NASH and NASH-related fibrosis include transaminases or GGT. .9:88-91 $^{-1}$

A second indication comes from the studies that did include a liver biopsy and hence a histological classification of NAFLD. These studies are summarised in Table 1. Matteoni et al. only found differences in liver-related mortality but not in all-cause or other cause mortality according to histological subtype of NAFLD..$^{92}$ Dam-Larsen et al. did not find differences in mortality comparing histologically proven patients with NAFL compared to the general population. ${ }^{93}$ However, more 
recent studies consistently show CVD being more prevalent in NAFLD patients, three out of four confining this risk to patients with $\mathrm{NASH} .{ }^{94-97}$ In the study examining prothrombotic factors, we also found an increase mainly in PAI-1 in association with more severe histological lesions, mainly confining the elevations in PAI-1 to patients with $\mathrm{NASH} .{ }^{74}$ Studies on levels of angiogenic factors in patients with NAFLD also showed the most pronounced changes in patients with NASH. ${ }^{9}$

Although these most recent data suggest that the risk is mainly associated with $\mathrm{NASH}$, or is at least more pronounced in patients with NASH compared with NAFLD, further methodologically stringent studies with long-term follow-up are needed to solve this question.

\section{Treatment of Non-alcoholic Fatty Liver Disease - Impact on Cardiovascular Disease?}

Currently there is no approved pharmacological treatment for NAFLD. ${ }^{12}$ Metformin and statins do not seem to improve liver histology, at least not in terms of fibrosis. ${ }^{98}$ Glitazones have a beneficial effect, as well as vitamin $\mathrm{E}$, but not in all patients. ${ }^{99-102}$ Lifestyle modification (diet and increased physical activity), if successful, improves NAFLD and also RouX-en-Y gastric bypass surgery ameliorates liver histology. ${ }^{11,12,103,104}$ Although it can be hypothesized that improving NAFLD reduces the risk of CVD, there is currently little data on potential changes in the risk of CVD in relation to the success of NAFLD treatment. Interestingly, two recent studies on the effects of statins on $C V$ events demonstrated a significantly more reduced $\mathrm{CV}$ event rate on statin treatment in patients with baseline elevation of liver tests (used as a surrogate marker for the presence of NAFLD), in relation to a significant improvement of liver tests in one study. ${ }^{105,106}$ The cardioprotective effect of statins was less pronounced in patients with normal liver tests at baseline. Glitazones also improve CV risk, but it is unclear to what extent this can be attributed to their beneficial effect on NAFLD. ${ }^{107-109}$ Furthermore, as outlined before, it is not clear whether the risk of CVD is increased in all subtypes of NAFLD. Therefore, no evidence-based recommendations can be formulated at present.

Nevertheless, it can be recommended to screen for NAFLD in every patient with risk factors for CVD or with established CVD, as well as to screen for CVD in every patient with NAFLD, and to treat accordingly with lifestyle modification. This recommendation is debated, as there are no data on cost-effectiveness and no pharmacological treatment when NAFLD is diagnosed. ${ }^{12}$ Metformin is frequently used, as it seems to have beneficial effects on CV risk, ${ }^{110-112}$ although also debated, ${ }^{113}$ in patients with insulin resistance. However, as outlined previously, metformin failed to show beneficial effects on liver histology. 2,98 Other metabolic factors should be treated according to the corresponding guidelines.

\section{Conclusion}

The role of NAFLD in the pathophysiology of CV abnormalities and hence its independent contribution to an increased risk of $\mathrm{CV}$ morbidity and mortality is increasingly evidenced by studies in animal models and by clinical data. Whether NAFL is still to be considered benign in this regard and whether the risk is hence confined to NASH is currently unclear but the risk seems at least to be more pronounced in NASH patients compared with NAFL. As the role of NAFLD in CVD becomes clearer, this aspect of NAFLD should probably be incorporated in the future guidelines on its treatment indications and paradigms.
1. Francque $S$, Non-alcoholic Fatty Liver Disease (NAFLD) and Non-alcoholic Steatohepatitis (NASH). In: van Damme P, Van Herck K, Michielsen P, et al., Chronic hepatitis and liver disease, oxford Textbook of Public Health, 5th edition, Oxford: Oxford University Press, 2009;1249-63.

2. Vernon G, Baranova A, Younossi ZM, Systematic review: the epidemiology and natural history of non-alcoholic fatty liver disease and non-alcoholic steatohepatitis in adults, Aliment disease and non-alcoholic steatoh

3. Verrijken A, Francque $S$, Van Gaal L, The metabolic syndrome and the liver, Acta Gastroenterol Belg, 2008;71(1):48-59.

4. Brunt EM, Janney CG, Di Bisceglie AM, et al., Nonalcoholic steatohepatitis: a proposal for grading and staging the histological lesions, Am J Gastroenterol, 1999;94(9):2467-74

5. Anstee QM, Targher G, Day CP, Progression of NAFLD to diabetes mellitus, cardiovascular disease or cirrhosis, Nat Rev Gastroenterol Hepatol, 2013;10(6):330-44.

6. Ballestri $\mathrm{S}$, Lonardo A Bonapace $\mathrm{S}$, et al., Risk of cardiovascular, cardiac and arrhythmic complications in patients with non-alcoholic fatty liver disease, World Gastroenterol, 2014;20(7):1724-45.

7. Brunt $E M$, Histopathology of non-alcoholic fatty liver disease Clin Liver Dis, 2009;13(4):533-44.

8. Begriche K, Massart J, Robin MA, et al., Drug-induced toxicity on mitochondria and lipid metabolism: mechanistic diversity and deleterious consequences for the liver, J Hepato 2011;54(4):773-94.

9. Kotronen A, Seppänen-Laakso T, Westerbacka J, et al., Comparison of lipid and fatty acid composition of the liver, subcutaneous and intra-abdominal adipose tissue, and subcutaneous and intra-abdominal adipose tissue,
serum, Obesity (Silver Spring), 2010:18(5):937-44.

10. Paradis V, Bedossa P, Definition and natural history of metabolic steatosis: histology and cellular aspects, Diabetes Metab, 2008;34(6 Pt 2):638-42

11. Ratziu V, Bellentani S, Cortez-Pinto $H$, et al., A position statement on NAFLD/NASH based on the EASL 2009 special conference, I Hepatol, 2010;53(2):372-84.

12. Chalasani N, Younossi Z, Lavine JE, et al., The diagnosis and management of non-alcoholic fatty liver disease: Practice guideline by the American Association for the Study of Liver Diseases, American College of Gastroenterology, and the American Gastroenterological Association, Am I Gastroenterol American Gastroenterol

13. Brunt EM, Liver biopsy diagnosis of hepatitis: clues to clinicallymeaningful reporting, Mo Med, 2010;107(2):113-8.

14. Bugianesi E, Leone $\mathrm{N}$, Vanni E, et al., Expanding the natural history of nonalcoholic steatohepatitis: from cryptogenic cirrhosis to hepatocellular carcinoma, Gastroenterology, 2002;123(1):134-40.
15. Musso G, Gambino R, Cassader M, Pagano G, Meta-analysis: natural history of non-alcoholic fatty liver disease (NAFLD) and diagnostic accuracy of non-invasive tests for liver disease severity, Ann Med, 2011;43(8):617-49.

16. Bugianesi E, Bellentani S, Bedogni $G$, et al., Clinical update on non-alcoholic fatty liver disease and steatohepatitis, Ann Hepatol, 2008;7(2):157-60

17. Yki-Järvinen $\mathrm{H}$, Non-alcoholic fatty liver disease as a cause and a consequence of metabolic syndrome, Lancet Diabetes andocrinol, 2014: pii: S2213-8587(14)70032-4.

18. Vanni $E$, Bugianesi $E$, Kotronen $A$, et al., From the metabolic syndrome to NAFLD or vice versa?, Dig Liver Dis, 2010;42(5):320-30

19. Tilg H, Moschen AR, Evolution of inflammation in nonalcoholic fatty liver disease: the multiple parallel hits hypothesis, Hepatology, 2010;52(5):1836-46.

20. Paradis V, Zalinski S, Chelbi E, et al., Hepatocellular carcinomas in patients with metabolic syndrome often develop without significant liver fibrosis: a pathological develop without significant liver fibrosis:
analysis, Hepatology, 2009:49(3):851-9.

21. Takuma Y, Nouso K, Nonalcoholic steatohepatitis-associated hepatocellular carcinoma: Our case series and literature review, World I Gastroenterol, 2010;16(12):1436-41.

22. Targher $\mathrm{G}$, Day CP, Bonora E, Risk of cardiovascular disease in patients with nonalcoholic fatty liver disease, $N$ Eng/ J Med, 2010;363(14):1341-50

23. Targher G, Bertolini L, Padovani R, et al., Associations between liver histology and carotid intima-media thickness in patients with nonalcoholic fatty liver disease, Arterioscler in patients with nonalcoholic fatty liver

24. Colak Y, Senates E, Yesil A, et al., Assessment of endothelial function in patients with nonalcoholic fatty liver disease, Endocrine, 2013;43(1):100-7.

25. Fracanzani AL, Burdick L, Raselli S, et al., Carotid artery intima-media thickness in nonalcoholic fatty liver disease Am J Med, 2008;121(1):72-8.

26. Villanova N, Moscatiello S, Ramilli S, et al., Endothelial dysfunction and cardiovascular risk profile in nonalcoholic fatty liver disease, Hepatology, 2005;42(2):473-80.

27. Pacifico L, Anania C, Martino $F$, et al., Functional and morphological vascular changes in pediatric nonalcoholic fatty liver disease, Hepatology, 2010;52(5):1643-51.

28. Brea A, Mosquera D, Martin E, et al., Nonalcoholic fatty liver disease is associated with carotid atherosclerosis: a case-control study, Arterioscler Thromb Vasc Biol, 2005;25(5):1045-50

29. Volzke H, Robinson DM, Kleine V, et al., Hepatic steatosis is associated with an increased risk of carotid atherosclerosis, World I Gastroenterol, 2005;11(12):1848-53.
30. Jung DH, Lee YJ, Ahn HY, et al., Relationship of hepatic steatosis and alanine aminotransferase with coronary calcification, Clin Chem Lab Med. 2010;48(12):1829-34.

31. Kim D, Choi SY, Park EH, et al., Nonalcoholic fatty liver disease is associated with coronary artery calcification, Hepatology, 2012;56(2):605-13.

32. Liu J, Musani SK, Bidulescu A, et al., Fatty liver, abdominal adipose tissue and atherosclerotic calcification in African Americans: the Jackson Heart Study, Atherosclerosis, 2012;224(2):521-5.

33. Sookoian S, Pirola CJ, Non-alcoholic fatty liver disease is strongly associated with carotid atherosclerosis: a systematic review, J Hepatol, 2008;49(4):600-7.

34. Yilmaz $\mathrm{Y}$, Kurt $\mathrm{R}$, Yonal $\mathrm{O}$, et al., Coronary flow reserve is impaired in patients with nonalcoholic fatty liver disease: association with liver fibrosis, Atherosclerosis, 2010;211(1):182-6.

35. Lautamäki R, Borra R, Iozzo P, et al., Liver steatosis coexists with myocardial insulin resistance and coronary dysfunction in patients with type 2 diabetes, Am I Physiol Endocrinol Metab. 2006;291(2):E282-90.

36. Nakamori S, Onishi K, Nakajima H, et al., Impaired myocardial perfusion reserve in patients with fatty liver disease assessed by quantitative myocardial perfusion magnetic resonance imaging, Circ J, 2012;76(9):2234-40

37. Açikel M, Sunay S, Koplay M, et al., Evaluation of ultrasonographic fatty liver and severity of coronary atherosclerosis, and obesity in patients undergoing coronary angiography, Anadolu Kardiyol Derg, 2009:9(4):273-9.

38. Wong VW, Wong GL, Yip GW, et al., Coronary artery disease and cardiovascular outcomes in patients with non-alcoholic fatty liver disease, Gut, 2011;60(12):1721-7.

39. Targher G, Pichiri I, Zoppini G, et al., Increased prevalence of cardiovascular disease in Type 1 diabetic patients with non-alcoholic fatty liver disease, J Endocrinol Invest, 2012;35(5):535-40

40. Targher G, Bertolini L, Padovani R, et al., Prevalence of non-alcoholic fatty liver disease and its association with cardiovascular disease in patients with type 1 diabetes, J Hepatol, 2010;53(4):713-8.

41. Treeprasertsuk S, Leverage S, Adams LA, et al., The Framingham risk score and heart disease in nonalcoholic fatty liver disease, Liver Int, 2012;32(6):945-50.

42. Stepanova M, Younossi ZM, Independent association between nonalcoholic fatty liver disease and cardiovascular disease in the US population, Clin Gastroenterol Hepatol, 2012;10(6):646-50.

43. Lazo M, Hernaez R, Bonekamp S, et al., Non-alcoholic fatty liver disease and mortality among US adults: prospective 
cohort study, BMJ, 2011:343:d6891

44. Perseghin $G$, Lattuada $G$, De Cobelli $F$, et al., Increased mediastinal fat and impaired left ventricular energy metabolism in young men with newly found fatty liver Hepatology, 2008;47(1):51-8

45. Bonapace S, Perseghin G, Molon G, et al., Nonalcoholic fatty liver disease is associated with left ventricular diastolic dysfunction in patients with type 2 diabetes, Diabetes Care, 2012;35(2):389-95

46. Rijzewijk LJ, Jonker JT, van der Meer RW, et al., Effects of hepatic triglyceride content on myocardial metabolism in type 2 diabetes, J Am Coll Cardiol, 2010;56(3):225-33.

47. Rijzewijk L, van der Meer RW, Smit JW, et al., Myocardial steatosis is an independent predictor of diastolic dysfunction in type 2 diabetes mellitus, I Am Coll Cardiol, 2008;52(22):1793-9.

48. Goland S, Shimoni S, Zornitzki T, et al., Cardiac abnormalities as a new manifestation of nonalcoholic fatty liver disease: echocardiographic and tissue Doppler imaging assessment J Clin Gastroenterol, 2006;40(10):949-55.

49. Mantovani A, Zoppini G, Targher G, et al., Non-alcoholic fatty liver disease is independently associated with left ventricula hypertrophy in hypertensive Type 2 diabetic individuals, I Endocrinol Invest, 2012:35(2):215-8.

50. Hallsworth $\mathrm{K}$, Hollingsworth KG, Thoma C, et al., Cardiac structure and function are altered in adults with nonalcoholic fatty liver disease, J Hepatol, 2013;58(4):757-62

51. Liu YC, Hung CS, Wu YW, et al., Influence of non-alcoholic fatty liver disease on autonomic changes evaluated by the time domain, frequency domain, and symbolic dynamics of heart rate variability, PLOS One, 2013;8(4):e61803.

52. Targher G, Mantovani A, Pichiri I, et al., Non-alcoholic fatty liver disease is associated with an increased prevalence of atrial fibrillation in hospitalized patients with type 2 diabetes, Clin Sci (Lond), 2013;125(6):301-9.

53. Sinner MF, Wang N, Fox CS, et al., Relation of circulating liver transaminase concentrations to risk of new-onset atria fibrillation, Am J Cardiol, 2013;111(2):219-24.

54. Targher G, Valbusa F, Bonapace S, et al., Association of nonalcoholic fatty liver disease with QTC interval in patients with type 2 diabetes, Nutr Metab Cardiovasc Dis, 2014;24(6):663-9.

55. Wannamethee SG, Whincup PH, Shaper AG, et al., Gammaglutamyltransferase, hepatic enzymes, and risk of incident heart failure in older men, Arterioscler Thromb Vasc Bio, 2012;32(3):830-5

56. Markus MR, Baumeister SE, Stritzke J, et al., Hepatic steatosis is associated with aortic valve sclerosis in the general population: the Study of Health in Pomerania (SHIP) Arterioscler Thromb Vasc Biol, 2013;33(7):1690-5.

57. Bonapace S, Valbusa F, Bertolini L, et al., Nonalcoholic fatty liver disease is associated with aortic valve sclerosis in patients with type 2 diabetes mellitus, PLOS One 2014:9(2): $\mathrm{e} 88371$.

58. Kim CH, Park JY, Lee KU, et al., Fatty liver is an independent risk factor for the development of Type 2 diabetes in Korean adults, Diabet Med, 2008;25(4):476-81.

59. Sung KC, Jeong WS, Wild SH, Byrne CD, Combined influence of insulin resistance, overweight/obesity, and fatty liver as risk factors for type 2 diabetes, Diabetes Care, 2012;35(4):717-22.

60. Bugianesi E, Moscatiello S, Ciaravella MF, Marchesini G, Insulin resistance in nonalcoholic fatty liver disease, Curr Pharm Des, 2010:16(17):1941-51.

61. Toledo FG, Sniderman AD, Kelley DE, Influence of hepatic steatosis (fatty liver) on severity and composition of dyslipidemia in type 2 diabetes, Diabetes Care, 2006;29(8):1845-50.

62. Adiels M, Westerbacka J, Soro-Paavonen A, et al., Acute suppression of VLDL1 secretion rate by insulin is associated with hepatic fat content and insulin resistance, Diabetologia, 2007;50(11):2356-65

63. Adiels M, Taskinen MR, Packard C, et al., Overproduction of large VLDL particles is driven by increased liver fat content in man, Diabetologia, 2006:49(4):755-65.

64. Lerman A, Zeiher AM, Endothelial function: cardiac events, Circulation, 2005;111(3):363-8

65. Schalkwijk CG, Stehouwer CD, vascular complications in diabetes mellitus: the role of endothelial dysfunction, Clin Sci (Lond), 2005; 109(2): 143-59.

66. Pasarin M, Abraldes JG, Rodriguez-Vilarrupla A, et al., Insulin resistance and liver microcirculation in a rat model of early
NAFLD, I Hepatol, 2011:55(5):1095-102.

67. Pasarin M, La Mura V, Gracia-Sancho J, et al., Sinusoida endothelial dysfunction precedes inflammation and fibrosis in a model of NAFLD, PLOS One, 2012;7(4):e32785.

68. Francque S, Laleman W, Verbeke L, et al., Increased intrahepatic resistance in severe steatosis: endothelia dysfunction, vasoconstrictor overproduction and altered microvascular architecture, Lab Invest, 2012;92(10):1428-39.

69. Coulon S, Francque S, Colle I, et al., Evaluation of inflammatory and angiogenic factors in patients with nonalcoholic fatty liver disease, Cytokine, 2012;59(2):442-9.

70. Coulon S, HeindryckX F, Geerts A et al., Angiogenesis in chronic liver disease and its complications, Liver int, 2011;31(2):146-62.

71. Khurana R, Simons M, Martin JF, Zachary IC, Role of angiogenesis in cardiovascular disease: a critical appraisal Circulation, 2005;112(12):1813-24.

72. Targher G, Chonchol M, Miele L, et al., Nonalcoholic fatty liver disease as a contributor to hypercoagulation and thrombophilia in the metabolic syndrome, Semin Thromb Hemost, 2009:35(3):277-87.

73. Van Gaal LF, Mertens IL, De Block CE, Mechanisms linking obesity with cardiovascular disease, Nature, 2006:444(7121):875-80

74. Verrijken A, Francque S, Mertens I, et al., Prothrombotic factors in histologically proven nonalcoholic fatty liver disease and nonalcoholic steatohepatitis, Hepatology, 2014;59(1):121-9.

75. Mertens I, Van Gaal LF, Visceral fat as a determinant of fibrinolysis and hemostasis, Semin Vasc Med, 2005;5(1):48-55.

76. Ruige JB, Ballaux DP, Funahashi T, et al., Resting metabolic rate is an important predictor of serum adiponectin concentrations: potential implications for obesity-related disorders, Am I Clin Nutr, 2005:82(1):21-5.

77. BruyndonckX L, Hoymans VY, Van Craenenbroeck AH, et al, Assessment of endothelial dysfunction in childhood obesity and clinical use, Oxid Med Cell Longev, 2013;2013:174782.

78. Bianchi G, Bugianesi E, Frystyk J, et al., Adiponectin soforms, insulin resistance and liver histology in nonalcoholic fatty liver disease, Dig Liver Dis, 2011;43(1):73-7.

79. Francque SM, Verrijken A, Mertens I, et al., Noninvasive assessment of nonalcoholic fatty liver disease in obese or overweight patients, Clin Gastroenterol Hepatol, 2012;10(10):1162-8.

80. Marra F, Bertolani C, Adipokines in liver diseases, Hepatology, 2009;50(3):957-69.

81. Ouedraogo R, Wu X, Xu SQ, et al., Adiponectin suppression of high-glucose-induced reactive oxygen species in vascular endothelial cells: evidence for involvement of a CAMP signaling pathway, Diabetes, 2006;55(6):1840-6.

82. Adams V, Heiker JT, Höllriegel R, et al., Adiponectin promotes the migration of circulating angiogenic cells through p38mediated induction of the CXCR4 receptor, Int I Cardiol, 2013;167(5):2039-46.

83. Lima-Cabello E, Garcia-Mediavilla MV, Miquilena-Colina ME, et al., Enhanced expression of pro-inflammatory mediators and liver $\mathrm{X}$-receptor-regulated lipogenic genes in nonalcoholic fatty liver disease and hepatitis C, Clin Sci (Lond) 2011;120(6):239-50

84. Espinola-Klein C, Gori T, Blankenberg S, Munzel T, Inflammatory markers and cardiovascular risk in the metabolic syndrome, Front Biosci (Landmark Ed), 2011:16:1663-74.

85. Pihlajamäki J Kuulasmaa T Kaminska D, et al. Serum interleukin 1 receptor antagonist as an independent marker of non-alcoholic steatohepatitis in humans, J Hepatol, 2012:56(3):663-70.

86. Mofrad $\mathrm{P}$, Contos MJ, Haque M, et al., Clinical and histologic spectrum of nonalcoholic fatty liver disease associated with normal ALT values, Hepatology, 2003;37(6):1286-92.

87. Suzuki A, Lymp J, Sauver JS, et al., Values and limitations of serum aminotransferases in clinical trials of nonalcoholic steatohepatitis, Liver Int, 2006;26(10):1209-16.

88. Bedogni G, Bellentani S, Miglioli L, et al., The Fatty Liver Index: a simple and accurate predictor of hepatic steatosis in the general population, BMC Gastroenterol, 2006;6:33.

89. Angulo P, Hui JM, Marchesini G, et al., The NAFLD fibrosis score: a noninvasive system that identifies liver fibrosis in patients with NAFLD, Hepatology, 2007;45(4):846-54.

90. Kotronen A, Peltonen M, Hakkarainen A, et al., Prediction of non-alcoholic fatty liver disease and liver fat using metabolic and genetic factors, Gastroenterology, 2009;137(3):865-72.
91. Younossi ZM, Jarrar M, Nugent C, et al., A novel diagnostic biomarker panel for obesity-related nonalcoholic steatohepatitis (NASH), Obes Surg, 2008;18(11):1430-7.

92. Matteoni CA, Younossi ZM, Gramlich T, et al., Nonalcoholic fatty liver disease: a spectrum of clinical and pathological severity, Gastroenterology, 1999;116(6):1413-9.

93. Dam-Larsen S, Franzmann M, Andersen IB, et al., Long term prognosis of fatty liver: risk of chronic liver disease and death, Gut, 2004;53(5):750-5

94. Adams LA, Lymp JF, St Sauver J, et al., The natural history of nonalcoholic fatty liver disease: a population-based cohort study, Gastroenterology, 2005;129(1):113-21.

95. Ekstedt M, Franzén LE, Mathiesen UL, et al., Long-term follow-up of patients with NAFLD and elevated liver enzymes, Hepatology, 2006;44(4):865-73

96. Rafiq N, Bai C, Fang $Y$, et al., Long-term follow-up of patients with nonalcoholic fatty liver, Clin Gastroenterol Hepatol 2009;7(2):234-8

97. Söderberg C, Stål P, Askling J, et al., Decreased survival of subjects with elevated liver function tests during a 28-year follow-up, Hepatology, 2010;51(2):595-602.

98. Musso G, Gambino R, Cassader M, Pagano G, A meta-analysis of randomized trials for the treatment of nonalcoholic fatty liver disease, Hepatology, 2010;52(1):79-104.

99. Ratziu V, Pienar L, Pharmacological therapy for non-alcoholic steatohepatitis: How efficient are thiazolidinediones? Hepatol Res, 2011;41(7):687-95

100. Ratziu V, Charlotte F, Bernhardt C, et al., Long-term efficacy of rosiglitazone in nonalcoholic steatohepatitis: results of the fatty liver improvement by rosiglitazone therapy (FLIRT 2) extension trial, Hepatology, 2010;51(2):445-53.

101. Sanyal AJ, Chalasani N, Kowdley KV, et al., Pioglitazone, vitamin $\mathrm{E}$, or placebo for nonalcoholic steatohepatitis, N Eng/ J Med, 2010;362(18):1675-85.

102. Mahady SE, Webster AC, Walker S, et al., The role of thiazolidinediones in non-alcoholic steatohepatitis - a systematic review and meta analysis, J Hepatol, 2011;55(6):1383-90.

103. Lassailly G, Caïazzo R, Pattou F, Mathurin P, Bariatric surgery for curing NASH in the morbidly obese?, J Hepatol, 2013;58(6):1249-51

104. Centis E, Marzocchi R, Suppini A, et al., The role of lifestyle change in the prevention and treatment of NAFLD, Curr Pharm Des, 2013:19(29):5270-9.

105. Athyros VG, Tziomalos K, Gossios TD, et al., Safety and efficacy of long-term statin treatment for cardiovascular events in patients with coronary heart disease and abnorma liver tests in the Greek Atorvastatin and Coronary Heart Disease Evaluation (GREACE) Study: a post-hoc analysis, Lancet, 2010;376(9756):1916-22.

106. Tikkanen MJ, Fayyad R, Faergeman O, et al., Effect of intensive lipid lowering with atorvastatin on cardiovascular outcomes in coronary heart disease patients with mild-tomoderate baseline elevations in alanine aminotransferase levels, Int J Cardiol, 2013;168(4):3846-52.

107. Genovese S, De Berardis G, Nicolucci A, et al., Effect of pioglitazone versus metformin on cardiovascular risk markers in type 2 diabetes, Adv Ther, 2013;30(2):190-202

108. Zou C, Hu H, Use of pioglitazone in the treatment of diabetes: effect on cardiovascular risk, Vasc Health Risk Manag 2013;9:429-33.

109. Vaccaro O, Masulli M, Bonora E, et al., Addition of either pioglitazone or a sulfonylurea in type 2 diabetic patients inadequately controlled with metformin alone: impact on cardiovascular events. A randomized controlled trial, Nutr Metab Cardiovasc Dis, 2012:22(11):997-1006.

110. Lexis $C P$, Wieringa WG, Hiemstra $B$, et al., Chronic metformin treatment is associated with reduced myocardial infarct size in diabetic patients with ST-segment elevation myocardial infarction, Cardiovasc Drugs Ther, 2014;28(2):163-71.

111. Lexis $C P$, van der Horst IC, Lipsic E, et al., Effect of metformin on left ventricular function after acute myocardia infarction in patients without diabetes: the GIPS-III randomized clinical trial, JAMA, 2014;311(15):1526-35.

112. Preiss D, Lloyd SM, Ford I, et al., Metformin for non-diabetic patients with coronary heart disease (the CAMERA study): a randomised controlled trial, Lancet Diabetes Endocrinol 2014;2(2):116-24.

113. Lexis CP, van der Horst IC, Metformin for cardiovascular disease: promise still unproven, Lancet Diabetes Endocrinol, 2014;2(2):94-5. 